\title{
LAGRANGIAN SYSTEMS IN THE PRESENCE OF SINGULARITIES
}

\author{
A. CAPOZZI, C. GRECO AND A. SALVATORE
}

(Communicated by George R. Sell)

\begin{abstract}
In this paper we study dynamical systems embedded in a conservative field of forces, whose potential is "singular." We look for $T$-periodic solutions of these systems by variational methods.
\end{abstract}

0. Introduction. In this paper we look for $T$-periodic solutions of the Lagrangian system of ordinary differential equations:

$$
\frac{d}{d t} \frac{\partial \mathcal{L}}{\partial \xi}(t, q, \dot{q})-\frac{\partial \mathcal{L}}{\partial q}(t, q, \dot{q})=0, \quad q \in C^{2}\left(\mathbf{R}, \mathbf{R}^{N}\right),
$$

where the Lagrangian function $\mathcal{L}(t, q, \xi)$ is given, as usual, by

$$
\begin{aligned}
& \mathcal{L}(t, q, \xi)=\frac{1}{2} \sum_{i, j=1}^{N} a_{i j}(t, q) \xi_{i} \xi_{j}+\sum_{i=1}^{N} b_{i}(t, q) \xi_{i}+c(t, q)-V(t, q), \\
& \qquad t \in \mathbf{R}, q, \xi \in \mathbf{R}^{N},
\end{aligned}
$$

and $a_{i j}(t, q), b_{i}(t, q), c(t, q), V(t, q)$ are $C^{1}$ real-valued functions, $T$-periodic in $t$. Moreover we suppose that the "potential" $V(t, q)$ is defined in $\mathbf{R} \times \Omega$, where $\Omega$ is an open subset of $\mathbf{R}^{N}$, and $V(t, q) \rightarrow-\infty$ as $q \rightarrow \partial \Omega$.

Many authors have studied this problem in the case when $\Omega=\mathbf{R}^{N}$ (so $\partial \Omega=\varnothing$ ) under various assumptions on the growth of $V(t, q)$ as $|q| \rightarrow \infty$ : cf., for instance, $[2,3,5,9,10]$. W. B. Gordon was the first to study our case by means of variational methods, and we refer to $[6,7]$ for the physical motivation of the problem (cf. also the end of this section). Finally we refer to $[1,8]$ for the case $V(t, q) \rightarrow+\infty$ as $q \rightarrow \partial \Omega\left(\Omega \neq \mathbf{R}^{N}\right)$.

In this paper we suppose that:

$(0.1)\left\{a_{i j}(t, q)\right\}_{i, j}$ is a symmetric matrix, and there exists a function $\lambda: \mathbf{R}^{N} \rightarrow$ ] $0,+\infty[$ such that:

(i) $\sum_{i, j=1}^{N} a_{i j}(t, q) \xi_{i} \xi_{j} \geq \lambda(q)|\xi|^{2}$ for any $t \in \mathbf{R}, q, \xi \in \mathbf{R}^{N}$;

(ii) there are real constants $c_{1}>0$ and $\nu \in\left[0,1\left[\right.\right.$ such that $\lambda(q) \geq c_{1}\left(|q|^{\nu}+1\right)^{-1}$ for any $q \in \mathbf{R}^{N}$.

(0.2) There exists $M>0$ such that $\left|b_{i}(t, q)\right| \leq M$ for any $i=1,2, \ldots, N$ and $t \in \mathbf{R}, q \in \mathbf{R}^{N}$.

(0.3) $c(t, q) \geq 0$ for any $t \in \mathbf{R}, q \in \mathbf{R}^{N}$.

Received by the editors December 30, 1985.

1980 Mathematics Subject Classification (1985 Revision). Primary 34C25; Secondary 58E05.

Work supported by G.N.A.F.A. of C.N.R. and by Ministero P.I. (40\%-60\%). 
(0.4) There exist a function $U \in C^{1}(\Omega, \mathbf{R})$, a neighborhood $\mathcal{N}$ of $\mathbf{R}^{N} \backslash \Omega$ and a constant $c_{2} \geq 0$ such that:

(i) $\lim _{q \rightarrow \partial \Omega} U(q)=-\infty$;

(ii) $-V(t, q) \geq\left|U^{\prime}(q)\right|^{2}-c_{2}$ for any $t \in \mathbf{R}$ and $q \in \mathcal{N} \cap \Omega$.

The theorems we now state were announced in rather different form in [4] (cf. Remark 0.2 for some generalizations).

THEOREM 0.1. Let $N=2$ and $\Omega=\mathbf{R}^{2} \backslash\{0\}$. Assume that (0.1)-(0.4) hold, and

$$
\begin{aligned}
& \text { there exist real constants } c_{3}, c_{4}>0 \text { and } \mu \in[0,2-\nu[ \\
& \text { such that } V(t, q) \leq c_{3}|q|^{\mu}+c_{4} \text { for any } t \in \mathbf{R}, q \in \Omega .
\end{aligned}
$$

Then there exists at least one $T$-periodic solution of (*).

THEOREM 0.2. Let $N \geq 2$, and let $\Omega$ be symmetric with respect to the origin. Assume that (0.1)-(0.5) hold, and

$$
a_{i j}, b_{i}, c, \text { and } V \text { are }(T / 2) \text {-periodic in } t
$$

$$
a_{i j}, c, \text { and } V \text { are even in } q \text { and } b_{i} \text { are odd in } q .
$$

Then there exists a pair $(q,-q)$ of $T$-periodic solutions of $(*)$.

REMARK 0.1. If $b_{i}=0$ and we deal with the autonomous case, then under the same hypotheses of Theorem 0.1 or 0.2 there exists a solution of $(*)$ with minimal period $T$ (cf. $\S 1$ below).

Notice that hypotheses $(0.1)$ and $(0.2)$ are motivated by the positiveness of the kinetic energy of a mechanical system; moreover we emphasize that $\left\{a_{i j}(t, q)\right\}_{i, j}$ can go to zero as $|q| \rightarrow \infty$. Condition (0.4) has been introduced by W. B. Gordon (cf. $[6,7])$; in [6] he considers the autonomous second-order Hamiltonian system: $\ddot{q}=-V^{\prime}(q)$ in the case when $V(q)$ is bounded from above as $|q| \rightarrow \infty$.

We observe that condition (0.5) permits one to consider, for instance, conservative dynamical systems with a periodic "forcing" term.

REMARK 0.2. As we shall see in the sequel, Theorem 0.1 still holds under more general assumptions on the open set $\Omega$ : for example, in the case when $\Omega=$ $\mathbf{R}^{N} \backslash P_{1} \cup P_{2}$, where $N>2$ and $P_{i}(i=1,2)$ are linear subspaces of $\mathbf{R}^{N}$ such that $P_{1} \cap P_{2}=\{0\}$ and $1 \leq \operatorname{dim}\left(P_{i}\right) \leq N-2, i=1,2$. This is the geometrical situation which occurs in the study of the planar $n$-body problem (cf. [6]).

Finally we give a simple application of Theorem 0.1 . Let us consider the system: $\ddot{q}=|q|^{-2}+f(t)$ where $f: \mathbf{R} \rightarrow \mathbf{R}^{2}$ is continuous and periodic. If we set $V(t, q)=$ $-|q|^{-2}+(f(t) \mid q)_{\mathbf{R}^{2}}$ then (0.1)-(0.5) are satisfied; (0.4) in particular, holds with $U(q)=\log |q|, \mathcal{N}=\left\{q \in \mathbf{R}^{2}|| q \mid<1\right\}$, and $c_{2}=\|f\|_{\infty}$.

1. Proofs of the theorems. If $x, y \in \mathbf{R}^{N}$, we denote by $|x|$ and $x y$ the Euclidean norm and the inner product in $\mathbf{R}^{N}$. For $1 \leq p \leq \infty$ let $\|q\|_{p}$ be the norm in $L^{p}\left(\mathbf{R}, \mathbf{R}^{N}\right)$; moreover we consider the Sobolev space $H^{1}=H^{1,2}\left([0, T], \mathbf{R}^{N}\right)$ obtained by the closure of the $C^{\infty} T$-periodic functions $q(t)$ with respect to the norm

$$
\|q\|=\left(\int_{0}^{T}|q(t)|^{2} d t+\int_{0}^{T}|\dot{q}(t)|^{2} d t\right)^{1 / 2} .
$$


$H^{1}$ is an Hilbert space, and we denote by $\langle\cdot, \cdot\rangle$ the duality between $H^{1}$ and its dual $H^{-1}$. We recall that $H^{1}$ is compactly embedded in $C\left([0, T], \mathbf{R}^{N}\right)$.

Finally, we set $\operatorname{Im}(q)=\{q(t) \mid t \in[0, T]\}$ and $\delta(q)=\sup _{x, y \in \operatorname{Im}(q)}|x-y|$.

Let us consider the functional:

$$
\begin{aligned}
f(q)= & \frac{1}{2} \int_{0}^{T} \sum_{i, j=1}^{N} a_{i j}(t, q) \dot{q}_{i} \dot{q}_{j} d t+\int_{0}^{T} \sum_{i=1}^{N} b_{i}(t, q) \dot{q}_{i} d t \\
& +\int_{0}^{T} c(t, q) d t-\int_{0}^{T} V(t, q) d t
\end{aligned}
$$

which is defined on the open subset $\Lambda^{1} \Omega \equiv\left\{q \in H^{1} \mid \operatorname{Im}(q) \subset \Omega\right\}$ of $H^{1}$. It is easy to verify that $f \in C^{1}\left(\Lambda^{1} \Omega, \mathbf{R}\right)$ and its critical points (that is, the zeros of $f^{\prime}$ ) are $T$-periodic solutions of $(*)$. The functional $f$ is, in general, not bounded from below on $\Lambda^{1} \Omega$; then, in order to prove Theorem 0.1 , we shall check that $f$ attains its minimum value on a nontrivial homotopy class of $\Lambda^{1} \Omega$, where $\Omega=\mathbf{R}^{2} \backslash\{0\}$.

We shall need the following lemma (in the sequel $a_{1}, a_{2}, \ldots$ will denote positive constants).

LEMMA 1.1. Let $\Omega=\mathbf{R}^{2} \backslash\{0\}$. Then $\|q\|_{\infty} \leq \delta(q)$ for any $q \in \Lambda^{1} \Omega$ which is not homotopic to a constant in $\Omega$.

PROOF. We argue by contradiction and assume that $\left|q\left(t_{0}\right)\right|>\delta(q)$ for some $t_{0} \in[0, T]$. Then $\operatorname{Im}(q)$ is contained in $\left\{x \in \mathbf{R}^{2}|| x-q\left(t_{0}\right) \mid \leq \delta(q)\right\} \subset \Omega$, and $q$ is homotopic to a constant in $\Omega$, so we get a contradiction.

REMARK 1.1. Since $\left|q\left(t_{1}\right)-q\left(t_{2}\right)\right|=\left|\int_{t_{1}}^{t_{2}} \dot{q}(t) d t\right| \leq\|\dot{q}\|_{2}\left|t_{1}-t_{2}\right|^{1 / 2}$, Lemma 1.1 implies: $\|q\| \leq c_{5}\|\dot{q}\|_{2}$, where $c_{5}>0$.

PROOF OF THEOREM 0.1. Let $\Lambda_{0}$ be a nontrivial homotopy class. In our assumptions, for any $q \in \Lambda_{0}$, we have

$$
\begin{aligned}
f(q)= & \frac{1}{2} \int_{0}^{T} \sum_{i, j=1}^{N} a_{i j}(t, q) \dot{q}_{i} \dot{q}_{j} d t+\int_{0}^{T} \sum_{i=1}^{N} b_{i}(t, q) \dot{q}_{i} d t \\
& +\int_{0}^{T} c(t, q) d t-\int_{0}^{T} V(t, q) d t \\
\geq & \frac{1}{2} \int_{0}^{T} \lambda(q)|\dot{q}|^{2} d t-M \int_{0}^{T}|\dot{q}| d t-\int_{0}^{T}\left(c_{3}|q|^{\mu}+c_{4}\right) d t \\
\geq & \frac{1}{2} \int_{0}^{T} c_{1}\left(|q|^{\nu}+1\right)^{-1}|\dot{q}|^{2} d t-M\|\dot{q}\|_{1}-c_{3}\|q\|_{\mu}^{\mu}-T c_{4} \\
\geq & \frac{c_{1}}{2}\left(a_{1}\|q\|^{\nu}+1\right)^{-1}\|\dot{q}\|_{2}^{2}-M\|\dot{q}\|_{1}-c_{3}\|q\|_{\mu}^{\mu}-T c_{4} \\
\geq & \frac{c_{1}}{2}\left(a_{1}\|q\|^{\nu}+1\right)^{-1} \frac{1}{c_{5}^{2}}\|q\|^{2}-a_{2}\|q\|-a_{3}\|q\|^{\mu}-T c_{4} \geq-a_{4}
\end{aligned}
$$

because $2-\nu>\max \{1, \mu\}$.

So the functional $f$ is bounded from below on $\Lambda_{0}$. Let $\left(q_{n}\right)_{n} \subset \Lambda_{0}$ be a minimizing sequence of $f$. From coercivity, it is bounded in $H^{1}$; then we can select a subsequence (still denoted by $\left.\left(q_{n}\right)_{n}\right)$ such that $q_{n} \rightarrow q_{0} \in H^{1}$ weakly in $H^{1}$ and 
uniformly. We shall prove that, if $q_{0} \in \partial \Lambda^{1} \Omega$, then we have

$$
\lim _{n \rightarrow \infty} \int_{0}^{T} V\left(t, q_{n}\right) d t=-\infty
$$

and this is not possible because $\left(f\left(q_{n}\right)_{n}\right)$ is bounded.

In fact, suppose $q_{0} \in \partial \Lambda^{1} \Omega$; since if $\operatorname{Im}\left(q_{0}\right) \subset \partial \Omega(1.1)$ is trivial, we can assume that $q_{0}(0) \in \partial \Omega$ and there exists $\tau>0$ such that $q_{0}(t) \in \mathcal{N} \cap \Omega$ for any $\left.\left.t \in\right] 0, \tau\right]$. Since $\left(\left\|\dot{q}_{n}\right\|_{2}\right)_{n}$ and $\left(U\left(q_{n}(\tau)\right)\right)_{n}$ are bounded, we have

$$
\begin{aligned}
-a_{5}-U\left(q_{n}(0)\right) & \leq U\left(q_{n}(\tau)\right)-U\left(q_{n}(0)\right) \\
& =\int_{0}^{\tau} \frac{d}{d t} U\left(q_{n}(t)\right) d t \leq \int_{0}^{\tau}\left|U^{\prime}\left(q_{n}\right)\right|\left|\dot{q}_{n}\right| d t \\
& \leq\left(\int_{0}^{\tau}\left|U^{\prime}\left(q_{n}\right)\right|^{2} d t\right)^{1 / 2}\left\|\dot{q}_{n}\right\|_{2} \leq a_{6}\left(\int_{0}^{\tau}\left|U^{\prime}\left(q_{n}\right)\right|^{2} d t\right)^{1 / 2},
\end{aligned}
$$

and therefore, by $(0.4)(\mathrm{i})$,

$$
\lim _{n \rightarrow \infty} \int_{0}^{\tau}\left|U^{\prime}\left(q_{n}\right)\right|^{2} d t=+\infty
$$

Since $V\left(t, q_{n}(t)\right) \leq a_{7}$ for any $t \in[0, T]$ and $n \in \mathrm{N}$, by (0.4)(ii) it follows that

$$
\begin{aligned}
\int_{0}^{T} V\left(t, q_{n}\right) d t & =\int_{0}^{\tau} V\left(t, q_{n}\right) d t+\int_{\tau}^{T} V\left(t, q_{n}\right) d t \\
& \leq-\int_{0}^{\tau}\left|U^{\prime}\left(q_{n}\right)\right|^{2} d t+\tau c_{2}+a_{7}(T-\tau)
\end{aligned}
$$

By the previous inequality and (1.2) we get (1.1).

We can conclude that $q_{0}$ belongs to $\Lambda^{1} \Omega$ and to $\Lambda_{0}$ also (by the uniform convergence). Moreover, by the weakly lower semicontinuity of $f$, it follows that $q_{0}$ is the minimum of $f$ on $\Lambda_{0}$.

Let us consider now the case $N \geq 2$ (as in Theorem 0.2). Since $\Omega$ can be homotopically trivial (for example if $\Omega=\mathbf{R}^{3} \backslash\{0\}$ ), we shall restrict the functional $f$ to the subspace (cf. [3])

$$
E=\left\{q \in H^{1} \mid q(t+T / 2)=-q(t)\right\} .
$$

Observe that each $q \in E$ has mean value zero; then Wirtinger's inequality holds:

$$
c_{6}\|q\|^{2} \leq\|\dot{q}\|_{2}^{2} \quad \text { for any } q \in E, \quad \text { where } c_{6}=2 \pi /(T+2 \pi) \text {. }
$$

The choice of the subspace $E$ is also motivated by the following lemma.

LEMMA 1.2. If $q \in E \cap \Lambda^{1} \Omega$ is a critical point of $f_{\mid E \cap \Lambda^{1} \Omega}$, then $q$ is a critical point of $f$.

PROOF. Let $q \in E \cap \Lambda^{1} \Omega$ be a critical point of $f_{\mid E \cap \Lambda^{1} \Omega}$, that is,

$$
\left\langle f^{\prime}(q), h\right\rangle=0 \text { for any } h \in E .
$$

We prove that (1.4) holds for any $h \in H^{1}$. In order to get this, fix $h \in H^{1}$ and set $h(t)=h_{1}(t)+h_{2}(t)$, where

$$
h_{1}(t)=h(t)-h(t+T / 2), \quad \text { and } \quad h_{2}(t)=h(t+T / 2) .
$$


Since $h_{1} \in E$, by (1.4), we have

$$
0=\left\langle f^{\prime}(q), h_{1}\right\rangle=\left\langle f^{\prime}(q), h\right\rangle-\left\langle f^{\prime}(q), h_{2}\right\rangle .
$$

By (0.6), (0.7) it is easy to verify that $\left\langle f^{\prime}(q), h_{2}\right\rangle=-\left\langle f^{\prime}(q), h\right\rangle$. Then the conclusion follows.

ProOF OF THEOREM 0.2. By Lemma 1.2 it suffices to look for the critical points of the functional $f$ on the open subset $E \cap \Lambda^{1} \Omega$ of $E$. By using (1.3) instead of Lemma 1.1, it can be seen, as in the proof of Theorem 0.1 , that $f$ is bounded from below and attains its minimum value.

Let us consider the autonomous case, and assume that $b_{i}(q)=0(i=1,2, \ldots, N)$. Moreover, suppose that $q$ is a critical point of $f$ and $T / k(k \in \mathbf{N}, k \geq 2)$ is a period of the function $q(t)$. Then it is easy to check that $q_{k}(t)=q(t / k)$ is still a critical point of $f$, and that $f\left(q_{k}\right)<f(q)$ (cf. [3]). Therefore $T$ is the minimal period of the solutions given by Theorems 0.1 and 0.2 .

Now we shall sketch some generalizations of Theorem 0.1 , as mentioned in Remark 0.2. Let $\Omega$ be an open set of $\mathbf{R}^{N}(N \geq 2)$. As in [6], we consider the following subset $\Lambda \subset \Lambda^{1} \Omega$ :

$q \in \Lambda \Leftrightarrow$ for any $c>0$ there exists a compact subset $K_{c}$ of $\mathbf{R}^{N}$ which contains any $p \in \Lambda^{1} \Omega$ such that $p$ is homotopic to $q$ in $\Omega$ and the arclength of $p$ is $\leq c$.

Clearly, if $\Omega=\mathbf{R}^{2} \backslash\{0\}$, then $q \in \Lambda \Leftrightarrow q$ is not homotopically trivial, but, in general, this is not the case.

Suppose $\Lambda \neq \varnothing$, and let $\Lambda_{0}$ be a homotopy class. Then we can minimize $f$ on $\Lambda_{0}$ provided a geometrical estimate like Lemma 1.1 is available for the functions in $\Lambda_{0}$. We limit ourself to stating the following variant of Lemma 1.1.

LEMMA 1.3. Let $\Omega=\mathbf{R}^{N} \backslash P_{1} \cup P_{2}$ when $P_{1}, P_{2}$ are as in Remark 0.2 .

Then $\Lambda \neq \varnothing$ and, if $\Lambda_{0}$ is a homotopy class $\subset \Lambda$, there exists $c_{7}>0$ such that, for any $q \in \Lambda_{0}$, we have $\|q\|_{\infty} \leq c_{7} \delta(q)$.

ProOF. It is easy to see that $\Lambda \neq \varnothing$; let $\Lambda_{0}$ be a homotopy class $\subset \Lambda$. Now, for any $r>0$, we set $\rho(r)=\sup \left\{\sigma>0 \mid\right.$ for any $x \in \mathbf{R}^{N}$ with $|x|=r, P_{1} \cap$ $B_{\sigma}(x)=\varnothing$ or $\left.P_{2} \cap B_{\sigma}(x)=\varnothing\right\}\left(B_{\sigma}(x) \equiv\left\{y \in \mathbf{R}^{N}|| y-x \mid<\sigma\right\}\right)$. Then $\rho(r)$ increases proportionally to $r$, that is, there exists $c_{8}>0$ such that $\rho(r)=c_{8} r$. Set $c_{7}=2 / c_{8}$, and suppose that there exists $q \in \Lambda_{0}$ such that $\|q\|_{\infty}>c_{7} \delta(q)$. Then $\|q\|_{\infty}=\left|q\left(t_{0}\right)\right|$ for some $t_{0} \in[0, T]$. Set $A=B_{\delta(q)}\left(q\left(t_{0}\right)\right)$ and observe that $\delta(q) \geq \rho\left(\left|q\left(t_{0}\right)\right|\right)$ (otherwise $P_{1} \cap A=\varnothing$ or $P_{2} \cap A=\varnothing$, and this implies $q \notin \Lambda_{0}$ ), so $\left|q\left(t_{0}\right)\right|=\|q\|_{\infty}>c_{7} \delta(q) \geq c_{7} \rho\left(\left|q\left(t_{0}\right)\right|\right)=c_{7} c_{8}\left|q\left(t_{0}\right)\right|=2\left|q\left(t_{0}\right)\right|$, which is not possible.

\section{REFERENCES}

1. V Benci, Normal modes of a Lagrangian system constrained in a potential well, Ann. Inst. H. Poincaré 1 (1984), 379-400.

2. V. Benci, A. Capozzi, and D. Fortunato, Periodic solutions of Hamiltonian systems with superquadratic potential, Ann. Mat. Pura Appl. 143 (1986), 1 46.

3. A. Capozzi, D. Fortunato, and A. Salvatore, Periodic solutions of Lagrangian systems with bounded potential, J. Math. Anal. Appl. 124 (1987), 482 494. 
4. A. Capozzi and A. Salvatore, Periodic solutions of Hamiltonian systems: The case of the singular potential, Nonlinear Functional Analysis and Applications (S. P. Singh, Editor), Reidel NATO AS1-e 173, 1986, 207-216.

5. F. Giannoni, Soluzioni periodiche di sistemi Hamiltoniani in presenza di vincoli, Pubbl. Dip. Mat. Univ. Pisa 11 (1983).

6. W. B. Gordon, Conservative dynamical systems involving strong forces, Trans. Amer. Math. Soc. 204 (1975), 113-135.

7. __ A minimizing property of Keplerian orbits, Amer. J. Math. 99 (1977), 961-971.

8. C. Greco, Remarks on periodic solutions of second order Hamiltonian systems in an unbounded potential well, Nonlinear Oscillation for Conservative Systems, Proc. Venice, January 1985 (A. Ambrosetti), Pitagora Ed., Bologna, 1985, pp. 37-41.

9. J. Mawhin and M. Willem, Multiple solutions of the periodic boundary value problem for some forced pendulum-type equations, J. Differential Equations 52 (1984), 264-287.

10. A. Salvatore, Periodic solutions of Hamiltonian systems with a subquadratic potential, Boll. Un. Mat. Ital. C 1 (1984), 393-406.

Dipartimento di Matematica, Università Degli Studi, Campus UniversiTARIO, 70125 BARI, ITALY 